\title{
A Semi-supervised Learning Framework to Cluster Mixed Data Types
}

\author{
Artur Abdullin \{ar.abdullin@louisville.edu\} and Olfa Nasraoui \{olfa.nasraoui@louisville.edu\} \\ Knowledge Discovery \& Web Mining Lab, Department of Computer Engineering and Computer Science, \\ University of Louisville, Louisville, KY
}

\begin{abstract}
Keywords:
semi-supervised clustering: mixed data type clustering
\end{abstract}

Abstract:

We propose a semi-supervised framework to handle diverse data formats or data with mixedtype attributes. Our preliminary results in clustering data with mixed numerical and categorical attributes show that the proposed semi-supervised framework gives better clustering results in the categorical domain. Thus the seeds obtained from clustering the numerical domain give an additional knowledge to the categorical clustering algorithm. Additional results show that our approach has the potential to outperform clustering either domain on its own or clustering both domains after converting them to the same target domain.

\section{Introduction}

Many algorithms exist for clustering. However most of them have been designed to optimally handle specific types of data, e.g the spherical kmeans was proposed to cluster text data (Dhillon and Modha, 2001). The algorithm in (Banerjee et al., 2005) has been designed for data with directional distributions that lie on the unit hypersphere such as text data. The k-modes has been designed specifically for categorical data (Huang, 1997). Special data types and domains have been also handled using specialized dissimilarity or distance measures. For example, the k-means, using the Euclidean distance, is optimal for compact globular clusters with numerical attributes.

Suppose that a data set comprises multiple types of data that can each be best clustered with a different specialized clustering algorithm or with a specialized dissimilarity measure. In this case, the most common approach has been to either convert all data types to the same type (e.g: from categorical to numerical or vice-versa) and then cluster the data with a standard clustering algorithm in that target domain; or to use a different dissimilarity measure for each domain, then combine them into one dissimilarity measure and cluster this dissimilarity matrix with an $O\left(N^{2}\right)$ algorithm.

To handle possibly diverse data formats and different sources of data, we propose a new approach for combining diverse representations or types of data. Examples of data with mixed attributes include network activity data (e.g. the KDD cup data), most existing census or demographic data, environmental data, and other scientific data. Our approach is rooted in SemiSupervised Learning (SSL), however it uses SSL in a completely novel way and for a new purpose that has never been the objective in previous SSL research and applications. More specifically, traditional semi-supervised learning or transductive learning has been used mainly to exploit additional information in unlabeled data to enhance the performance of a classification model (traditionally trained using only labeled data) (Zhu et al., 2003), or to exploit some external supervision in the form of a few labeled data to improve the results of clustering unlabeled data. However, in this paper, we will use SSL "without" any external labels. Rather, the helpful labels will be "inferred" from multiple Unsupervised Learners (UL), such that each UL transmits to the other UL, a subset of confident labels that it has learned on its own from the data in one domain, along with some of the data that has been labeled with these newly discovered (cluster) labels. Hence the ULs from the different domains try to guide each other using mutual semi-supervision.

The rest of this paper is organized as follows. Section 2 gives an overview of related work. Sec- 
tion 3 presents our proposed framework to cluster mixed and multi-source data. Section 4 evaluates the proposed approach and Section 5 presents the conclusions of our preliminary investigation.

\section{Related Work}

Many semi-supervised algorithms have been proposed (Zhong, 2006) including co-training (Blum and Mitchell, 1998), the transductive support vector machine (Joachims, 1999), entropy minimization (Guerrero-Curieses and Cid-Sueiro, 2000), semi-supervised Expectation Maximization (Nigam et al., 2000), graph-based approaches (Blum and Chawla, 2001; Zhu et al., 2003), and clustering-based approaches (Zeng et al., 2003). In semi-supervised clustering, labeled data can be used in the form of (1) initial seeds (Basu et al., 2002), (2) constraints (Wagstaff et al., 2001), or (3) feedback (Cohn et al., 2003). All these existing approaches are based on modelbased clustering (Zhong and Ghosh, 2003) where each cluster is represented by its centroid. Seedbased approaches use labeled data only to help initialize cluster centroids, while constrained approaches keep the grouping of labeled data unchanged throughout the clustering process, and feedback-based approaches start by running a regular clustering process and finally adjusting the resulting clusters based on labeled data.

Most successful clustering algorithms are specialized for specific types of attributes. For instance, categorical attributes have been handled using specialized algorithms such as k-modes, ROCK or CACTUS. The main idea of the $\mathrm{k}-$ modes is to select $k$ initial modes, followed by allocating every object to the nearest mode (Huang, 1997). The k-modes algorithm uses the match dissimilarity measure to measure the distance between categorical objects (Kaufman and Rousseeuw, 1990). ROCK is an adaptation of an agglomerative hierarchical clustering algorithm, which heuristically optimizes a criterion function defined in terms of the number of "links" between transactions or tuples, defined as the number of common neighbors between them. Starting with each tuple in its own cluster, they repeatedly merge the two closest clusters until the required number of clusters remain (Guha et al., 2000). The central idea behind CACTUS is that a summary of the entire data set is sufficient to compute a set of "candidate" clusters which can then be validated to determine the actual set of clusters. The CACTUS algorithm consists of three phases: computing the summary information from the data set, using this summary information to discover a set of candidate clusters, and then determining the actual set of clusters from the set of candidate clusters (Ganti et al., 1999). The spherical k-means algorithm is a variant of the k-means algorithm that uses the cosine similarity instead of the Euclidean distance. The algorithm computes a disjoint partition of the document vectors and for each partition, computes a centroids that is then normalized to have unit Euclidean norm (Dhillon and Modha, 2001). This algorithm was successfully used for clustering transactional or text (text documents are often represented as sparse high-dimensional vectors) data. Numerical data has been clustered using k-means, DBSCAN and many other algorithms. The k-means algorithm is a partitional or non-hierarchical clustering method, designed to cluster numerical data in which each cluster has a center called mean. The k-means algorithm operates as follow: starting with a specified number $k$ of initial cluster centers, the remaining data is reallocated or assigned, such that each data point is assigned to the nearest cluster. This is continued with repeatedly recomputing the new centers of the data assigned to each cluster and changing the membership assignments of the data points to belong to the nearest cluster until the objective function (which is the sum of distance values between the data and the assigned cluster's centroids), centroids or membership of the data points converge (MacQueen, 1967). DBSCAN is a density-based clustering algorithm designed to discover arbitrarily shaped clusters. A point $x$ is directly density reachable from a point $y$ if it is not farther than a given distance $\epsilon$ (i.e., it is part of its $\epsilon$-neighborhood), and if the $\epsilon$-neighborhood of $y$ has more points than an input parameter $N_{\text {min }}$ such that one may consider $y$ and $x$ to be part of a cluster (Ester et al., 1996).

The above approaches have the following limitations:

- Specialized clustering algorithms can fall short when they must handle different data types.

- Data type conversion can result in the loss of information or the creation of artifacts in the data.

- Different data sources may be hard to combine for the purpose of clustering because of the problem of duplication of data and the problem of missing data from one of the sources, in addition to the problem of heterogeneous 
types of data from multiple sources.

Algorithms for mixed data attributes exist, for instance the k-prototypes (Huang, 1998) and INCONCO algorithms (Plant and Böhm, 2011). The k-prototypes algorithm integrates the kmeans and the k-modes algorithms to allow for clustering objects described by mixed numerical and categorical attributes. The k-prototypes works by simply combining the Euclidean distance and the categorical (matching) distance measures in a weighted sum. The choice of the weight parameter and the weighting contribution of the categorical versus numerical domains cannot vary from one cluster to another, and this can be a limitation for some data sets. The INCONCO algorithm extends the Cholesky decomposition to model dependencies in heterogeneous data and, relying on the principle of Minimum Description Length, integrates numerical and categorical information in clustering. The limitations of INCONCO include that it assumes a known probability distribution model for each domain, and it assumes that the number of clusters is identical in both the categorical and the numerical domains. It is also limited to two domains.

Our proposed approach is reminiscent of ensemble-based clustering (Al-Razgan and Domeniconi, 2006; Ghaemi et al., 2009). However, one main distinction is that our approach enables the different algorithms running in each domain to reinforce or supervise each other during the intermediate stages, until the final clustering is obtained. In other words, our approach is more collaborative. Ensemble-based methods, on the other hand, were not intended to provide a collaborative exchange of knowledge between different data "domains," while the individual algorithms are still running, but rather to combine the end results of several runs, several algorithms, and so on.

\section{Semi-supervised Framework for Clustering Mixed Data Types}

Our proposed semi-supervised framework can use specifically designed clustering algorithms which can be distinct and specialized for the following different types of data, however all the algorithms are bound together within a collaborative scheme:

1. For categorical data types, the algorithms kmodes (Huang, 1997), ROCK (Guha et al.,
2000), CACTUS (Ganti et al., 1999), etc, can be used.

2. For transactional or text data, the spherical k-means algorithm (Dhillon and Modha, 2001), or any specialized algorithm can be used

3. For numerical data types, one can use the kmeans (MacQueen, 1967), DBSCAN (Ester et al., 1996), etc.

4. For graph data, one can use KMETIS (Karypis and Kumar, 1998), spectral clustering (Shi and Malik, 2000), etc.

In the following sub-sections, we distinguish between two cases depending on whether the number of clusters is the same across the different domains of the data.

\subsection{The Case of an Equal Number of Clusters in Each Data Type or Domain}

Our initial implementation reported in this paper, can handle data records composed of two parts: numerical and categorical, within a semisupervised framework that consists of the following stages:

1. The first stage consists of dividing the set of attributes into two subsets: one subset, called domain $T_{1}$, with only attributes of numerical type (age, income, etc), and another subset, called domain $T_{2}$, with attributes of categorical type (eyes color, gender, etc).

2. The next stage is to cluster each subset using a specifically designed algorithm for that particular data type. In our experiments, we used k-means (MacQueen, 1967) for numerical type attributes $T_{1}$, and k-modes (Huang, 1997) for categorical type attributes $T_{2}$. Both algorithms start from the same random initial seeds and run for a small number of iterations $\left(t_{n}\right.$ and $t_{c}$ for $\mathrm{k}$-means and k-modes, respectively), yielding (data-cluster) membership matrices $M_{T_{1}}$ and $M_{T_{2}}$, respectively.

3 . In the third stage, we compare the cluster centroids obtained in the first domain, $T_{1}$ and the second domain, $T_{2}$ and find the best combination of both for each of the domains. First, we solve a cluster correspondence problem between the two domains using the Hungarian method (Frank, 2005; Kuhn, 1955) using as weight matrix, the entry-wise reciprocal of the Jaccard coefficient matrix, which is computed using the cluster memberships $M_{T_{1}}$ and $M_{T_{2}}$ of the $T_{1}$ and $T_{2}$ domains respectively. Then using the membership matrices $M_{T_{1}}$ and $M_{T_{2}}$, 
we compute the Davies-Bouldin (DB) indices $d b_{M_{T_{1}}}^{T_{1}}$ and $d b_{M_{T_{2}}}^{T_{1}}$ (Davies and Bouldin, 1979) in data domain $T_{1}$ for each cluster centroid obtained respectively, from clustering the data in domain $T_{1}$ and from clustering the data in domain $T_{2}$ from the previous stage (2). Similarly, we also compute the DB indices $d b_{M_{T_{1}}}^{T_{2}}$ and $d b_{M_{T}}^{T_{2}}$ in data domain $T_{2}$ for each cluster centroid obtained respectively, from clustering the data in domain $T_{1}$ and from clustering the data in domain $T_{2}$. Note that computing a DB index for every cluster centroid is essentially the same as computing the original overall DB index but without taking the sum over all centroids. To find the best combination of centroids for domain $T_{1}$, we compare $d b_{M_{T_{1}}}^{T_{1}}$ and $d b_{M_{T_{2}}}^{T_{1}}$ for each centroid resulting from clustering the data in domain $T_{1}$ and resulting from clustering the data in domain $T_{2}$, and then take only those centroids which score a lower value in the DB index, thus forming better clusters in one domain compared to the other. We then perform a similar operation for domain $T_{2}$. The outputs of this stage are two sets, each consisting of the best combination of cluster centroids or prototypes for each of the data domains $T_{1}$ and $T_{2}$, respectively.

4. In this stage, we use the best seeds obtained from stage 3 to recompute the cluster centroids in the first domain by running k-means for a small number $\left(t_{n}\right)$ of iterations; then compare these recomputed centroids against the cluster centroids that were computed in the second domain in the previous iteration (as explained in detail in stage 3 ) and find the best cluster centroids' combination for the second domain $\left(T_{2}\right)$.

5. Here, we use the best seeds obtained from stage 4 to initialize the k-modes algorithm in domain $T_{2}$, and run it for $t_{c}$ iterations. Then again, we compare these recomputed centroids against the cluster centroids computed in the first domain in the previous iteration (as explained in detail in stage 3 ) and find the best cluster centroids' combination for the first domain $\left(T_{1}\right)$.

6. We repeat stages 4 and 5 until both algorithms converge or the number of exchange iterations exceeds a maximum number. The general flow of our approach is presented in Figure 1.

We compared the proposed mixed-type clustering approach with the following two classical baseline approaches for clustering mixed numerical and categorical data.

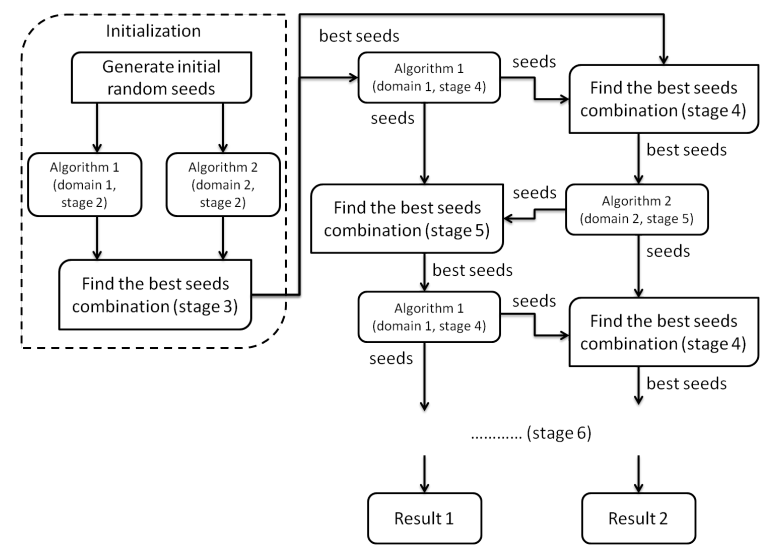

Figure 1: Overview of the semi-supervised seeding clustering approach.

Baseline 1: Conversion: The first baseline approach is to convert all data to the same attribute type and cluster it. We call this method the conversion algorithm. Since we have attributes of two types, there are two options to perform this algorithm:

1. Convert all numerical type attributes to categorical type attributes and run k-modes.

2. Convert all categorical type attributes to numerical type attributes and run k-means.

Baseline 2: Splitting: The second classical baseline approach is to run k-means and k-modes independently on the numerical and categorical subsets of attributes, respectively. We call this method the splitting algorithm.

The conversion algorithm requires data type conversion: from numerical to categorical and from categorical to numerical. There are several ways to convert a numerical type attribute $z$, ranging in $\left[z_{\min }, z_{\max }\right]$, to a categorical type attribute $y$, also known as "discretization" (Gan et al., 2007):

(i) by mapping the $n$ numerical values, $z_{i}$, to $N$ categorical values $y_{i}$ using direct categorization. Then the categorical value is defined as $y_{i}=\left\lfloor\frac{N\left(z_{i}-z_{\min }\right)}{\left(z_{\max }-z_{\min }\right)}\right\rfloor+1$, where \lfloor\rfloor denotes the largest integer less than or equal to $z$. Obviously, if $z_{i}=z_{\max }$, we get $y_{i}=N+1$, and we should set $y_{i}=N$.

(ii) by mapping the $n$ numerical values to $N$ categorical values using a histogram binning based method.

(iii) by clustering the $n$ numerical values into $N$ clusters using any numerical clustering algorithm (e.g. k-means). The optimal number of clusters $N$ can be chosen based on some validation criterion. 
In the current implementation, we use clusterbased conversion (iii) with the Silhouette index as a validity measure because this results in the best conversion. There are also several methods to convert categorical type attributes to numerical type attributes:

(i) by mapping the $n$ values of a nominal attribute to binary values using 1-of- $n$ encoding, resulting into transactional-like data, with each nominal value becoming a distinct binary attribute

(ii) by mapping the $n$ values of an ordinal nominal attribute to integer values in the range of 1 to $n$, resulting in numerical data with $n$ values

(iii) without any mapping, but instead modifying our distance measure so that for those nominal attributes, a suitable deviation is computed (e.g. a match between two categories results in a distance of 0 , while a non-match results in a maximal distance of 1 ). In this case, we need a clustering algorithm that works on the distance matrix instead of the input data vectors (also called relational clustering ).

In our implementation, we used the first method (1-of- $n$ encoding).

\subsection{The Case of a Different Number of Clusters or Different Cluster Partitions in each Data Type or Domain}

In our preliminary design above, the number of clusters is assumed to be the same in each domain. This can be considered as the default approach, and has the advantage of being easier to design. However, in real life data, there are two challenges:

- Case 1: The first challenge is when each data domain naturally gives rise to a different number of clusters, which is simple to understand.

- Case 2: The second challenge is when regardless of whether the number of clusters are similar or different in the different domains, their nature is actually completely different, and this will be illustrated with the following example.

How do we combine the results of clustering in different domains if the numbers of clusters are different? Let us look at the example shown in Figure 2, which for visualization purposes, artificially splits the two numerical features into two distinct domains, thus illustrating the difficulties with mixed domains. Here we have two domains or (artificially different) data types $T_{1}$ and $T_{2}$. In total, taking into account both data domains or

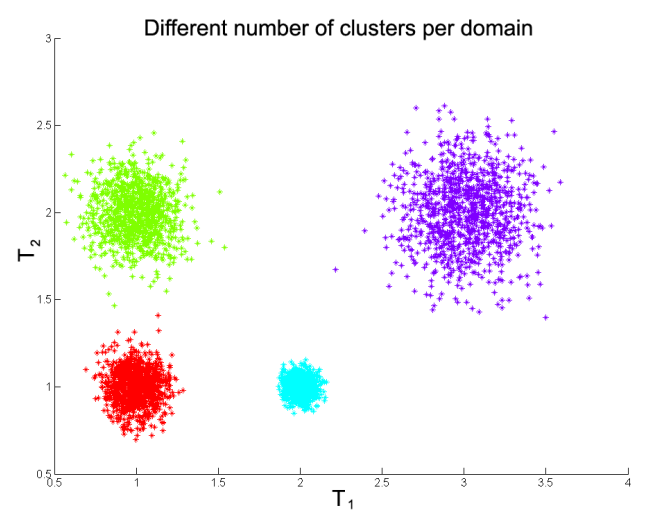

Figure 2: Different number of clusters per domain. types $T_{1}$ and $T_{2}$, we have four distinct clusters, however if we cluster each domain separately, we see that in $T_{1}$, we have three clusters, while in $T_{2}$, we have only two clusters. This illustrates Case 2 and gives rise to the problem of judiciously combining the clustering results emerging from each domain into a coherent clustering result with correct cluster labelings for all the data points.

We propose the following algorithm to cluster such a data set, that we emphasize, actually targets completely different data domains or types that cannot be compared using traditional attribute-based distance measures.

1. First, cluster $T_{1}$ with $k_{T_{1}}$ number of clusters and cluster $T_{2}$ with $k_{T_{2}}$ number of clusters. Let $M_{T_{1}}$ be the cluster membership matrix of domain $T_{1}$ and $M_{T_{2}}$ be the cluster membership matrix of domain $T_{2}$. Therefore, $M_{T_{1}}$ is an $n \times k_{T_{1}}$ matrix and $M_{T_{2}}$ is an $n \times k_{T_{2}}$ matrix, where $n$ is the number of data records. The membership matrix $M_{T}$ is such that entry $M_{T}[i, j]$ is 1 or 0 depending on whether or not point $i$ belongs to cluster $j$ in the current domain $T$.

2. Next, match each cluster $j_{1}$ in $T_{1}$ to the corresponding cluster $j_{2}$ in $T_{2}$. For this purpose, we compute the Jaccard coefficient matrix $J$ of size $k_{T_{1}} \times k_{T_{2}}$ in which entry $J\left[j_{1}, j_{2}\right]$ is defined as follows:

$$
J\left[j_{1}, j_{2}\right]=\frac{\left|C_{T_{1}, j_{1}} \cap C_{T_{2}, j_{2}}\right|}{\left|C_{T_{1}, j_{1}} \cup C_{T_{2}, j_{2}}\right|},
$$

where $C_{T, j}$ is the set of points that belong to cluster $j$ in domain $T$, i.e.,

$$
C_{T, j}=\left\{x_{i} \mid M_{T}(i, j)>0\right\} .
$$

Then we compute the optimal cluster correspondence using the Hungarian method with the weight matrix $W$ in which every entry, $W\left[j_{1}, j_{2}\right]=1 / J\left[j_{1}, j_{2}\right]$ (Kuhn, 1955; Frank, $2005)$. 
3. Finally, merge the clustering results of domains $T_{1}$ and $T_{2}$ using Algorithm 1. Let $T_{\max }$ be the domain with the highest number of clusters $k_{T_{\max }}=\max \left\{k_{T_{1}}, k_{T_{2}}\right\}$ and $M_{T_{\max }}$ be the membership matrix of that domain. Let $T_{\text {other }}$ be the other domain with a number of clusters $k_{T_{\text {other }}}\left(k_{T_{\text {other }}} \leq k_{T_{\text {max }}}\right)$ and let its membership matrix be $M_{T_{o t h e r}}$.

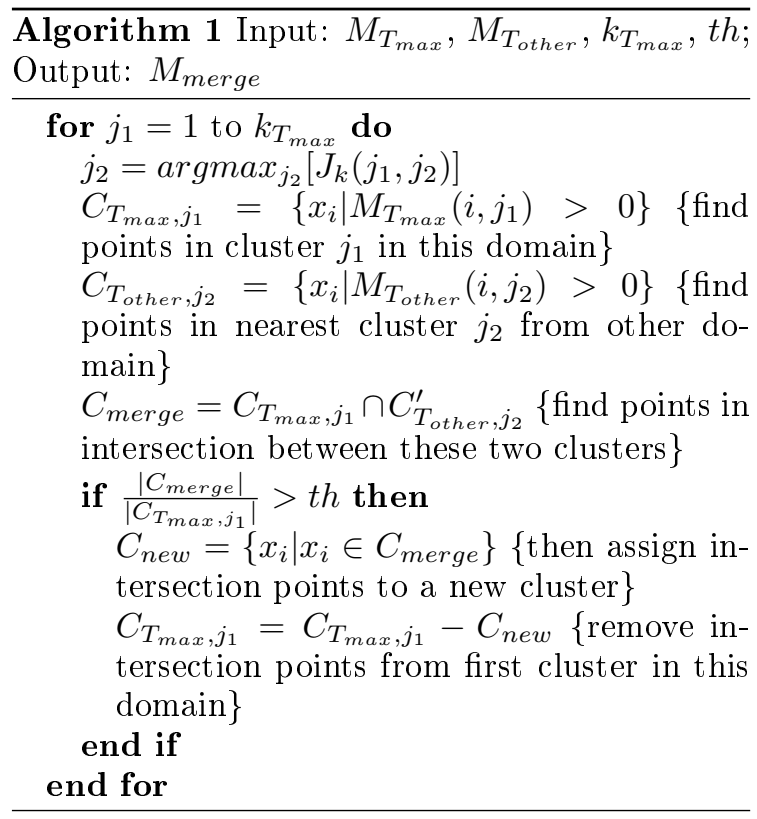

\subsection{Computational Complexity}

The complexity of the proposed approach is mainly determined based on the complexity of the embedded base algorithms used in each domain. In addition, there is the overhead complexity resulting from the coordination and alternating seed exchange process between the different domains during the mutual supervision process. The main overhead computation in the latter step is the cluster matching, validity scoring and comparison performed in stage 3 (which is then repeatedly invoked at the end of the subsequent stages 4 and 5). Stage 3 involves the following computations: first, the computation of the Jaccard coefficient matrix using the cluster memberships of the domains in time $O\left(k^{2} N\right)$ (assuming the number of clusters to be of similar order $k$ ), then, solving the correspondence problem between the two domains using the Hunguarian method in time $O\left(k^{3}\right)$, and finaly, computating of the DB indices for each cluster centroid in both domains in time $O\left(k^{2} N\right)$. Thus, the total overhead complexity of stage 3 is $O\left(k^{2} N\right)$ since $k \ll N$. With the k-means and k-modes as the base algorithms, the total computational complexity of the proposed approach is $O(N)$.

\section{Experimental Results}

\subsection{Clustering Evaluation}

The proposed semi-supervised framework was evaluated using several internal and external clustering validity metrics. Note that in calculating all internal indices that require a distance measure, we used the square of the Euclidean distance for numerical data types and the simple matching distance (Kaufman and Rousseeuw, 1990) for categorical data types.

- Internal validity metrics

- The Davies-Bouldin (DB) index is a function of the ratio of the sum of withincluster scatter to between-cluster separation (Davies and Bouldin, 1979). Hence the ratio is small if the clusters are compact and far from each other. That is, the DB index will have a small value for a good clustering.

- The Silhouette index is calculated based on the average silhouette width for each sample, average silhouette width for each cluster and overall silhouette width for the entire data set (Rousseeuw, 1987). Using this approach, each cluster can be represented by its silhouette, which is based on the comparison of its tightness and separation. A silhouette value close to 1 means that the data sample is well-clustered and assigned to an appropriate cluster. A silhouette value close to zero means that the data sample could be assigned to another cluster, and the data sample lies halfway between both clusters. A silhouette value close to -1 means that the data sample is misclassified and is located somewhere in between the clusters.

- The Dunn index is based on the concept of cluster sets that are compact and well separated (Dunn, 1974). The main goal of this measure is to maximize the inter-cluster distances and minimize the intra-cluster distances. A higher value of the Dunn index mean a better clustering.

- External validity metrics

- Purity is a simple evaluation measure that assumes that an external class label is 
Table 1: Data sets properties.

\begin{tabular}{|c|c|c|c|}
\hline Data set & $\begin{array}{c}\text { No. of } \\
\text { Records }\end{array}$ & $\begin{array}{c}\text { No. of Numerical } \\
\text { Attributes }\end{array}$ & $\begin{array}{c}\text { No. of Categorical } \\
\text { Attributes }\end{array}$ \\
\hline \hline Adult & 45179 & 6 & 8 \\
\hline Heart Disease Data & 303 & 6 & 7 \\
\hline Credit Approval Data & 690 & 6 & 9 \\
\hline
\end{tabular}

available to evaluate the clustering results. First, each cluster is assigned to the class which is most frequent in that cluster, then the accuracy of this assignment is measured by the ratio of the number of correctly assigned data samples to the number of data points. A bad clustering has a purity close to 0 , and a perfect clustering has a purity of 1 . Purity is very sensitive to the number of clusters, in particular, purity is 1 if each point gets its own cluster (Manning et al., 2008).

- Entropy is a commonly used information theoretic external validation measure that measures the purity of the clusters with respect to given external class labels (Xiong et al., 2006). A perfect clustering has an entropy close to 0 which means that every cluster consists of points with only one class label. A bad clustering has an entropy close to 1 .

- Normalized mutual information (NMI) estimates the quality of the clustering with respect to a groundtruth class membership (Strehl et al., 2000). It measures how closely a clustering algorithm could reconstruct the underlying label distribution in the data. The minimum NMI is 0 if the clustering assignment is random with respect to true class membership. The maximum NMI is 1 if the clustering algorithm could perfectly recreate the true class memberships.

\subsection{Equal Number of Clusters in Each Data Type or Domain}

\subsubsection{Real data sets}

We experimented with three real-life data sets with the characteristics shown in Table 1. All three data sets were obtained from the UCI Machine Learning Repository (Frank and Asuncion, 2010).

- Adult Data. The adult data set was extracted by Barry Becker from the 1994 Census database. The data set has two classes: People who make over $\$ 50 \mathrm{~K}$ a year and people who make less than $\$ 50 \mathrm{~K}$. The original data set consists of 48,842 instances. After deleting instances with missing and duplicate attributes we obtained 45, 179 instances.

- Heart Disease Data. The heart disease data, generated at the Cleveland Clinic, contains a mixture of categorical and numerical features. The data comes from two classes: people with no heart disease and people with different degrees of heart disease.

- Credit Approval Data. The data set has 690 instances, which were classified in two classes: approved and rejected.

\subsubsection{Results with the real data sets.}

Since all three data sets have two classes, we clustered them in two clusters. ${ }^{1}$ We repeated each experiment 50 times (10 times for the larger adult data set), and report the mean, standard deviation, minimum, median, and maximum values for each validation metric (in the format of mean \pm std [ min, median, max ]).

- Adult Data: Table 2 shows the results of the adult data set using the semi-supervised framework, the conversion algorithm, and the splitting algorithm, with the best results in a bold font. As the table illustrates, the semisupervised framework performs better in both domains: showing significant improvements in DB and Silhouette indices for the numerical domain and almost all validity indices for the categorical domain. Note the low minimum value of the $\mathrm{DB}$ and high maximum value of the Silhouette indices in the numerical domain, showing that over all runs, the proposed semi-supervised approach could achieve a better clustering than classical baseline approaches.

- Heart Disease Data: Table 3 shows the results of clustering the heart disease data set using the three approaches. The conversion algorithms yielded better clustering results for the numerical domain based on the Dunn index and all external indices. The semisupervised approach outperforms the conversion algorithm in the categorical domain but conceded to the splitting algorithm.

- Credit Approval Data: Table 4 shows the results of clustering the credit approval data set. Again, the semi-supervised approach outperforms the traditional algorithms for the categorical type attributes based on the internal indices but concedes to the splitting algorithm

\footnotetext{
${ }^{1}$ We realize the possibility of more than one cluster per class, however we defer such an analysis to the future.
} 
Table 5: Basic characteristics of the Iris data set.

\begin{tabular}{|c|c|c|c|c|c|}
\hline Attribute & Min & Max & Mean & Standard deviation & Class Correlation (Pearson's CC) \\
\hline \hline Sepal length & 4.3 & 7.9 & 5.84 & 0.83 & 0.7826 \\
\hline Sepal width & 2.0 & 4.4 & 3.05 & 0.43 & -0.4194 \\
\hline Petal length & 1.0 & 6.9 & 3.76 & 1.76 & 0.9490 \\
\hline Petal width & 0.1 & 2.5 & 1.20 & 0.76 & 0.9565 \\
\hline
\end{tabular}

in terms of all external indices One possible reason is that the cluster structure does not match the "true" class labels or ground truth, which is common in unsupervised learning. The splitting algorithms yielded better clustering results for the numerical domain based on the DB and Silhouette indices. Also note the low minimum value of the $\mathrm{DB}$ and high maximum value of the Silhouette indices in the numerical domain for the semi-supervised approach, showing that this approach can win by a large margin, and this is one of the further areas of focus for our ongoing work. Trying to reach these best results in an unsupervised way.

\subsection{Different Number of Clusters or Different Cluster Partitions in each Data Type or Domain}

\subsubsection{Data sets}

In the following experiments, as we have done with our illustrating example above, we validate our second algorithm on the data sets satisfying Case 2 (described in Section 3.2) of non-coherent cluster partitions across the different domains. Although in the Iris data set, the attributes are of the same type, we artificially split them into two domains so that we can validate the method and visualize the input data and the results. The validity of this example can generalize for different domains, because we do not exploit any attribute-based distance measure between the different data domains (as would be the case for really different domains). The Iris data set is a benchmark set that contains 3 classes of 50 data instances each, where each class refers to a type of iris plant: iris Setosa, iris Versicolour, iris Virginica.

\subsubsection{Experiments}

All three experiments were performed on the Iris data set. We repeated each experiment 10 times and report only the best results. For validation purposes, we used the class labels. For class cluster assignment we used the Jaccard coefficient, meaning that a class will be assigned to the cluster with highest Jaccard coefficient.
Table 6: Overview of the experiments.

\begin{tabular}{|c|c|c|c|c|c|c|c|}
\hline Experiment Number & Data set & $T_{1}$ & $T_{2}$ & $k_{T_{1}}$ & $k_{T_{2}}$ & $K$ & Threshold \\
\hline \hline 1 & Iris & $\{1\}$ & $\{3\}$ & 2 & 3 & 3 & 0.6 \\
\hline 2 & Iris & $\{2\}$ & $\{4\}$ & 2 & 3 & 3 & 0.6 \\
\hline 3 & Iris & $\{1,3\}$ & $\{2,4\}$ & 2 & 3 & 3 & 0.6 \\
\hline
\end{tabular}

Table 7: Experiment 1: Evaluation measures for the semi-supervised merging algorithm and k-means (bold results are best).

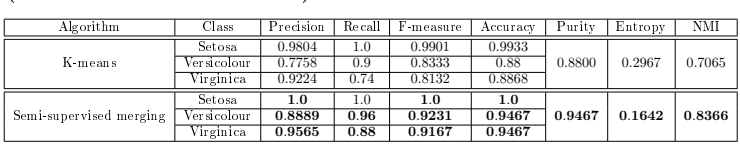

Experiment 1 We first take only the first and third features of the IRIS data set. Let $T_{1}$ be the sepal length (first feature) and $T_{2}$ be the petal length (third feature). We chose those two features because the first feature has a low class correlation index while the third feature has a high class correlation index. The first experiment was performed in the following steps:

1. Cluster the data in domain $T_{1}$ using k-means $(k=2)$

2. Cluster the data in domain $T_{2}$ using k-means $(k=3)$

3. Merge the two clustering results using the merging algorithm described in Algorithm 1. Notice that one of the output parameters is $K$, which is the number of clusters after merging. We set the overlap threshold parameter to $t h=0.6$.

4. Compare this result with the clustering result of k-means using $k=K$ clusters, performed on domains $T_{1}$ and $T_{2}$ together.

Table 7 shows the results in terms of classification accuracy, precision, recall, F-measure, purity, entropy and NMI for each algorithm (the results in bold are better).

Experiment 2 We next performed a similar experiment to the above but this time, domain $T_{1}$ is the 2 nd feature - sepal width, while $T_{2}$ is the 4 th feature - petal width. The overlap threshold was set to $t h=0.6$. On the Table 8 shown the results for the second experiment in terms of relevance measures.

Experiment 3 We repeated the same experiment as above, but this time, domain $T_{1}$ consists of the 1 st and 3 rd features, while $T_{2}$ consists of Table 8: Experiment 2: Evaluation measures for the semi-supervised merging algorithm and k-means (bold results are best).

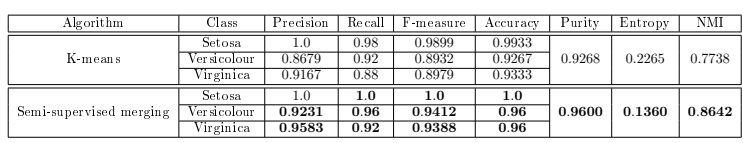


Table 2: Clustering result for the adult data set.

\begin{tabular}{|c|c|c|c|c|c|c|}
\hline Algorithm & \multicolumn{2}{|l|}{ Semi-supervised } & \multicolumn{2}{|l|}{$\mathrm{Co}$} & \\
\hline Data type & Numerical & Categorical & Numerical & Categorical & Numerical & Categorical \\
\hline "DB Index & $\pm 1.09[0.42,3.44,3.77]$ & $\overline{ \pm 0.14[1.10,1.1}$ & $\pm 7.70[1.48,12.3$ & $1.50 \pm 0.23[1.22,1.4$. & 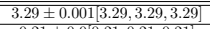 & 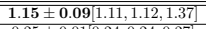 \\
\hline Silh. In & $0.29 \pm 0.18[0.18,0.21,0.71]$ & & $0.07 \pm 0.05$ & 0.2 & 0.21 & 0.2 \\
\hline Dunn Index & $1 e-4 \pm 4.2 e-4[1.2 e-5,1.2 e-5,1.1 e-3]$ & $0.125 \pm 0.0[0.12$ & $0 \pm 0.00[0.00,0.00,0.00]$ & $0.00 \pm 0.00[0.00,0.00,0$ & $0 \pm 0.00[0.00,0.00,0.00]$ & $0.125 \pm 0[0.125,0.125,0.125]$ \\
\hline "Puri & $\overline{\overline{0.62 \pm 0.070 .52 .0}}$ & 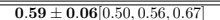 & $\overline{\overline{0.62 \pm 0.110 .}}$ & $0.56 \pm 0$. & $\overline{0.64 \pm 0}$ & 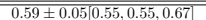 \\
\hline Entro & $0.77 \pm 0.03[0.72,0.78,0.79]$ & $0.73 \pm 0.6$ & $0.73 \pm 0.06[0.69,0.69,0.8$ & $0.73 \pm 0.02[0.70,0.74,0.75]$ & $0.71 \pm 0.00[0.71,0.71,0.71]$ & $0.73 \pm 0.01[0.71,0.73,0.73]$ \\
\hline NMI & $0.06 \pm 0.03[0.02,0.05,0.10]$ & $0.09 \pm 0.001[0.08,0.09,0.11]$ & $0.08 \pm 0.07[2.1 e-4,0.13, \mathbf{0 . 1 3}]$ & $0.09 \pm 0.02[0.07,0.08,0.12]$ & $0.10 \pm 0.00[0.10,0.10,0.10]$ & $0.09 \pm 0.01[0.08,0.08,0.11]$ \\
\hline
\end{tabular}

Table 3: Clustering result for the heart disease data set.

\begin{tabular}{|c|c|c|c|c|c|c|}
\hline Algorithm & \multicolumn{2}{|l|}{ Semi- } & \multicolumn{2}{|l|}{ Con } & \\
\hline Data type & Numerical & Categorical & Numerical & Categorical & Numerical & Categorical \\
\hline DB Index & 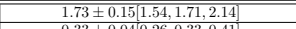 & $0.80 \pm 0.17[0.65,0.77,1.42]$ & 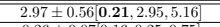 & 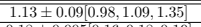 & $\overline{1.65} \pm \mathbf{0 . 0 0 3}[1.65,1.65,1.65]$ & $\begin{array}{l}.75,0.75,0.77] \\
\end{array}$ \\
\hline Silh. Index & $0.04[0.26,0.33,0.41]$ & & $0.26 \pm 0.07$ & $0.18 \pm 0.005[0.16,0$. & $\mathbf{0 . 3 6} \pm \mathbf{0 . 0 0 5}[0.36,0.36,0.36]$ & $0.31 \pm 0.00$ \\
\hline $\begin{array}{l}\text { Dunn Index } \\
\end{array}$ & $3.3 e-3 \pm 2.2 e-3[1.2 e-5,2.3 e-4,0.35]$ & $\mathbf{0 . 1 4} \pm \mathbf{0}[0.14,1$ & $\mathbf{0 . 0 4} \pm \mathbf{0 . 1 4}[0.015,0$ & $0.13 \pm 0.04[0.07,0$. & $4.6 e-3 \pm 0[4.6 e-3,4.6 e-3,4.6 e-3]$ & $\mathbf{0 . 1 4} \pm 0[0.14,0.14,0.14]$ \\
\hline Purity & $\overline{0.03[0 .}$ & 0.78 & & 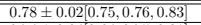 & $\frac{7.75,0.75]}{2}$ & $0.81 \pm$ \\
\hline Entropy & $0.84 \pm 0.03[0.79,0.84,0.91]$ & $0.74 \pm 0.04$ & $\mathbf{0 . 7 2} \pm \mathbf{0 . 1 1}[0.67,0.67,0.99]$ & $0.74 \pm 0$ & $0.80 \pm 0.003[0.80$, & $\mathbf{0 . 7 1} \pm \mathbf{0 . 0 2}[0.69,0.69,0.76]$ \\
\hline NMI & $0.15 \pm 0.03[0.08,0.16,0.20]$ & $0.25 \pm 0.04[0.13,0.24,0.30]$ & $\mathbf{0 . 2 8} \pm \mathbf{0 . 1 1}[2.1 e-4,0.32,0.32]$ & $0.26 \pm 0.04[0.18,0.25,0.36]$ & $0.19 \pm 0.004[0.18,0.19,0.19]$ & $0 . \mathbf{2 9} \pm \mathbf{0 . 0 2}[0.23,0.30,0.30]$ \\
\hline
\end{tabular}

Table 9: Experiment 3: Evaluation measures for the semi-supervised merging algorithm and k-means (bold results are best).

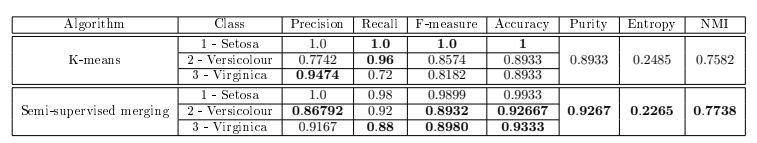

the 2 nd and 4 th features. The overlap threshold was set to $t h=0.6$. The results of the second experiment are shown in Table 9 .

\subsubsection{Results with the IRIS data set}

In the first and second experiments, the semisupervised merging algorithm outperformed the $\mathrm{k}$-means algorithm. In the third experiment, the merging algorithm obtained similar results to the k-means algorithm, although it still outperformed the k-means in terms of the purity of the results and giving a lower entropy of the clustering overall.

\section{Conclusions}

The results of our preliminary study show that the proposed semi-supervised framework tends to yield better clustering results in the categorical domain. Thus the seeds obtained from clustering the numerical domain tend to provide additional helpful knowledge to the categorical clustering algorithm (in this case, the K-modes algorithm). This information is in turn used to avoid local minima and obtain a better clustering in the categorical domain. We are currently completing our study by (1) extending our experiments and methodology to mixed data involving transactional information (particularly text and clickstreams) as one of the types, and (2) devising a suitable method for further combining the results of the multiple clusterings performed on each data type or domain. This is because, although each one of the data type-specific algo- rithms receives some guidance from the algorithm that clustered the other data types, the final results are currently not combined, but are rather still being evaluated in each domain separately. One promising direction is to combine the multiple clustering results such that the best clustering decisions are selected (or merged) from each result. A challenging issue is whether to merge decisions at the cluster prototype/parameter level or at the data partitioning/labeling level, or both. We are also extending our technique so that the number of clusters is allowed to vary from one domain (or attribute type) to another.

\section{Acknowledgment}

This work was supported by US National Science Foundation Data Intensive Computation Grant IIS-0916489.

\section{REFERENCES}

Al-Razgan, M. and Domeniconi, C. (2006). Weighted clustering ensembles. In Proc. of the 6th SIAM $I C M L$

Banerjee, A., Dhillon, I. S., Ghosh, J., Sra, S., and Ridgeway, G. (2005). Clustering on the unit hypersphere using von mises-fisher distributions. Journal of ML Research, 6.

Basu, S., Banerjee, A., and Mooney, R. (2002). Semisupervised clustering by seeding. In Proc. of 19 th $I C M L$.

Blum, A. and Chawla, S. (2001). Learning from Labeled and Unlabeled Data Using Graph Mincuts. In Proc. 18th ICML, pages 19-26.

Blum, A. and Mitchell, T. (1998). Combining labeled and unlabeled data with co-training. In Proc. of the 11th annual conference on $C L$ theory, pages 92-100.

Cohn, D., Caruana, R., and Mccallum, A. (2003). Semi-supervised clustering with user feedback. Technical report. 
Table 4: Clustering result for the credit card data set.

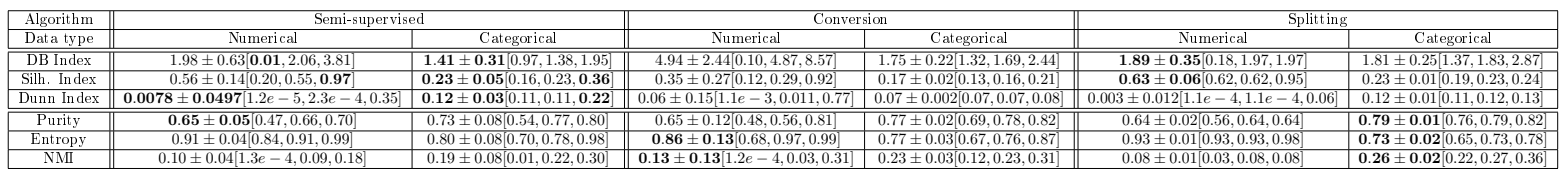

Davies, D. L. and Bouldin, D. W. (1979). A cluster separation measure. Pattern Analysis and $M a$ chine Intelligence, pages 224-227.

Dhillon, I. S. and Modha, D. S. (2001). Concept decompositions for large sparse text data using clustering. Mach. Learn., 42:143-175.

Dunn, J. C. (1974). Well separated clusters and optimal fuzzy partitions. J. Cybern, 4:95-104.

Ester, M., peter Kriegel, H., S, J., and Xu, X. (1996). A density-based algorithm for discovering clusters in large spatial databases with noise. In Proc. of the Second International Conference on $K D D$, pages $226-231$.

Frank, A. (2005). On kuhn's hungarian method - a tribute from hungary. Naval Research Logistics (NRL), 52:2-5.

Frank, A. and Asuncion, A. (2010). UCI machine learning repository.

Gan, G., Ma, C., and Wu, J. (2007). Data Clustering: Theory, Algorithms, and Applications. Society for Industrial and Applied Mathematics.

Ganti, V., Gehrke, J., and Ramakrishnan, R. (1999). Cactus - clustering categorical data using summaries. In Proc. of the 5th ACM SIGKDD International Conference on KDD, pages 73-83.

Ghaemi, R., Sulaiman, M. N., Ibrahim, H., and Mustapha, N. (2009). A survey: Clustering ensembles techniques.

Guerrero-Curieses, A. and Cid-Sueiro, J. (2000). An entropy minimization principle for semisupervised terrain classification. In Image Processing, 2000 International Conference on, volume 3, pages $312-315$.

Guha, S., Rastogi, R., and Shim, K. (2000). Rock: A robust clustering algorithm for categorical attributes. Information Systems, 25:345 - 366.

Huang, Z. (1997). A fast clustering algorithm to cluster very large categorical data sets in data mining. In In Research Issues on KDD, pages 1-8.

Huang, Z. (1998). Extensions to the k-means algorithm for clustering large data sets with categorical values. Data Mining and Knowledge Discovery, 2:283-304.

Joachims, T. (1999). Transductive inference for text classification using support vector machines. In Proc. of 16th ICML, pages 200-209, Bled, SL.

Karypis, G. and Kumar, V. (1998). A fast and high quality multilevel scheme for partitioning irregular graphs. SIAM Journal on Scientific Comp., 20(1):359-392.

Kaufman, L. and Rousseeuw, P. (1990). Finding Groups in Data An Introduction to Cluster Analysis.
Kuhn, H. W. (1955). The hungarian method for the assignment problem. Naval Research Logistic Quarterly, 2:83-97.

MacQueen, J. B. (1967). Some methods for classification and analysis of multivariate observations. In Proc. of the 5th Berkeley Symposium on Math. Statistics and Probability, volume 1, pages 281297.

Manning, C. D., Raghavan, P., and Schtze, H. (2008). Introduction to Information Retrieval.

Nigam, K., McCallum, A. K., Thrun, S., and Mitchell, T. (2000). Text classification from labeled and unlabeled documents using em. Mach. Learn., 39:103-134.

Plant, C. and Böhm, C. (2011). Inconco: interpretable clustering of numerical and categorical objects. In Proc. of the 17th ACM SIGKDD International Conference on KDD, pages 11271135.

Rousseeuw, P. (1987). Silhouettes: a graphical aid to the interpretation and validation of cluster analysis. J. Comput. Appl. Math., 20:53-65.

Shi, J. and Malik, J. (2000). Normalized cuts and image segmentation. IEEE Transactions on Pattern Analysis and Machine Intelligence, 22(8):888-905.

Strehl, A., Strehl, E., Ghosh, J., and Mooney, R. (2000). Impact of similarity measures on webpage clustering. In Workshop on AI for Web Search, pages 58-64.

Wagstaff, K., Cardie, C., Rogers, S., and Schrödl, S. (2001). Constrained k-means clustering with background knowledge. In Proc. of the 18th ICML, pages $577-584$.

Xiong, H., Wu, J., and Chen, J. (2006). Kmeans clustering versus validation measures: a data distribution perspective. In Proc. of the 12th ACM SIGKDD international conference on $K D D$, pages 779-784.

Zeng, H.-J., Wang, X.-H., Chen, Z., Lu, H., and Ma, W.-Y. (2003). Cbc: clustering based text classification requiring minimal labeled data. In Data Mining, Third IEEE ICDM, pages $443-450$.

Zhong, S. (2006). Semi-supervised model-based document clustering: A comparative study. Mach. Learn., 65:3-29.

Zhong, S. and Ghosh, J. (2003). A unified framework for model-based clustering. Journal of $M L$ Research, 4:1001-1037.

Zhu, X., Ghahramani, Z., and Lafferty, J. (2003). Semi-supervised learning using gaussian fields and harmonic functions. In Proc. 20th International Conf. on $M L$, pages 912-919. 